\title{
Multi-Modality Imaging of a Case of Multiple Intra-Cardiac Aneurysms Complicated by Mitral Valve Leaflet Perforation
}

\section{Ruchika Meel, PhD ${ }^{1^{*}}$, Liam Lorentz, FCRAD ${ }^{2}$, Ricardo Goncalves, MMED ${ }^{3}$ and Cert. in Cardiology (SA), Bongane Ngutshane, MD ${ }^{4}$ and Shungu Mogaladi, MMED ${ }^{4}$ (cardio-thoracic surgery)}

\author{
${ }^{1}$ Division of Cardiology, Department of Internal Medicine, Chris Hani Baragwanath Hospital, \\ University of the Witwatersrand, South Africa \\ ${ }^{2}$ Department of Radiology, Chris Hani Baragwanath Hospital, South Africa \\ ${ }^{3}$ Department of Cardiology, Olivedale Hospital, South Africa \\ ${ }^{4}$ Division of Cardio-thoracic Surgery, Department of General Surgery, Charlotte Maxeke Hospital, \\ University of the Witwatersrand, South Africa
}

*Corresponding author: Ruchika Meel, MBChB, MMED, Cert. in Cardiology, PhD, FEACVI, Division of Cardiology, Department of Internal Medicine, Chris Hani Baragwanath Academic Hospital, University of the Witwatersrand, 26 Chris Hani Road, Johannesburg, 1864, South Africa, Tel: +27-721702227

\section{Keywords \\ Heart failure, Multi-modality imaging, Mitral valve, Intra-car- diac aneurysms, Dyspnea}

\section{Introduction}

Intra-Cardiac aneurysms are a rare entity [1]. In the past case reports and series have described the entity of "annular sub-valvular left ventricular aneurysms" in patients of African descent [2]. These authors proposed the aforementioned aneurysms to be of congenital aetiology. Majority of the time the aneurysms were located in the sub-mitral or sub-aortic positions and were diagnosed by echocardiography and ventriculography. Multiple discrete left ventricular aneurysms complicated by mitral valve perforation and incompetence, in the absence of coronary artery disease is a unique presentation. Herein, we describe this uncommon case with the aid of two and three dimensional echocardiography and Cardiac Computed Tomography.

\section{Case}

A 38-year-old African Male with no significant past medical history presented to a peripheral Hospital in early March 2019 with symptoms of weight loss, fa- tigue, fever, night sweats, pleuritic chest pains and cough productive of yellow sputum of about two weeks duration. There was a history of tuberculosis contact at home. At this presentation clinical exam revealed a blood pressure of $92 / 62 \mathrm{mmHg}$, heart rate of 77 beats per minute, Respiratory rate of 35 breaths/min with Oxygen saturation of $96 \%$. On general exam there was pallor of nails and conjunctiva and peripheral oedema. On systemic exam he was noted to have percussion dullness in the region of middle lobe of the left lung and decrease air entry was noted. There were no crackles reported. Cardiovascular system exam revealed cardiomegaly with displaced apex beat in the $6^{\text {th }}$ intercostal space mid-axillary line with grade $4 / 6$ pan-systolic murmur in the mitral area. Blood chemistry revealed low Haemoglobin of $9.8 \mathrm{~g} / \mathrm{dl}$ secondary to iron deficiency, normal white cell count and a raised C-reactive protein at $33 \mathrm{mg} / \mathrm{l}$ and normal urea and electrolytes. Klebsiella pneumoniae and Citrobacter Freundii were noted on sputum microscopy, culture and sensitivity. He was treated with Ceftriaxone and Gentamicin for community acquired pneumonia, and subsequently discharged with out-patient cardiac clinic date. He presented again to the same Hospital in late September 2019, where an assessment of congestive cardiac failure was made sec-

Citation: Meel R, Lorentz L, Goncalves R, Ngutshane B, Mogaladi S (2020) Multi-Modality Imaging of a Case of Multiple Intra-Cardiac Aneurysms Complicated by Mitral Valve Leaflet Perforation. Int J Clin Cardiol 7:199. doi.org/10.23937/2378-2951/1410199

Accepted: September 01, 2020; Published: September 03, 2020

Copyright: (c) 2020 Meel R, et al. This is an open-access article distributed under the terms of the Creative Commons Attribution License, which permits unrestricted use, distribution, and reproduction in any medium, provided the original author and source are credited. 
ondary to severe mitral regurgitation due to posterior mitral leaflet prolapse. He was treated with diuretics and transfused blood for anaemia. He had a C-reactive protein of $29 \mathrm{mg} / \mathrm{l}$. All blood cultures were negative. He was subsequently referred to the Cardiologist at Chris Hani Baragwanath Hospital for possibility of mitral valve replacement.

Upon arrival to the cardiology clinic, the patient was noted to be hemodynamically stable with a blood pressure of $126 / 83 \mathrm{mmHg}$ and heart rate of 88 beats/min. He was not in heart failure. He had a displaced, volume overloaded apex beat and a grade $5 / 6$ pan-systolic mitral regurgitation murmur. Twelve lead electrocardiograms showed sinus rhythm with wide $P$ wave suggestive of left atrial enlargement and early repolarisation. His blood parameters revealed improved Haemoglobin at $11.1 \mathrm{~g} /$ dl, CRP of $37 \mathrm{mg} / \mathrm{l}$ and a normal urea and electrolytes. He underwent trans-thoracic echocardiography (TTE), which revealed a left ventricular end-diastolic diameter of $62 \mathrm{~mm}$ and left ventricular end- systolic diameter of $34.7 \mathrm{~mm}$ with an EF of $65 \%$. Right ventricular systolic function was preserved with a trans-annular planar systolic excursion of $2.6 \mathrm{~cm}$ and RV S' velocity of $15.5 \mathrm{~cm} / \mathrm{s}$. There was severe eccentric mitral regurgitation and severe tricuspid regurgitation with an estimated pulmonary artery systolic pressure of $82 \mathrm{mmHg}$. No masses or vegetation's were noted. Two discrete aneurysms were noted, one just below the mitral annulus inferior to the

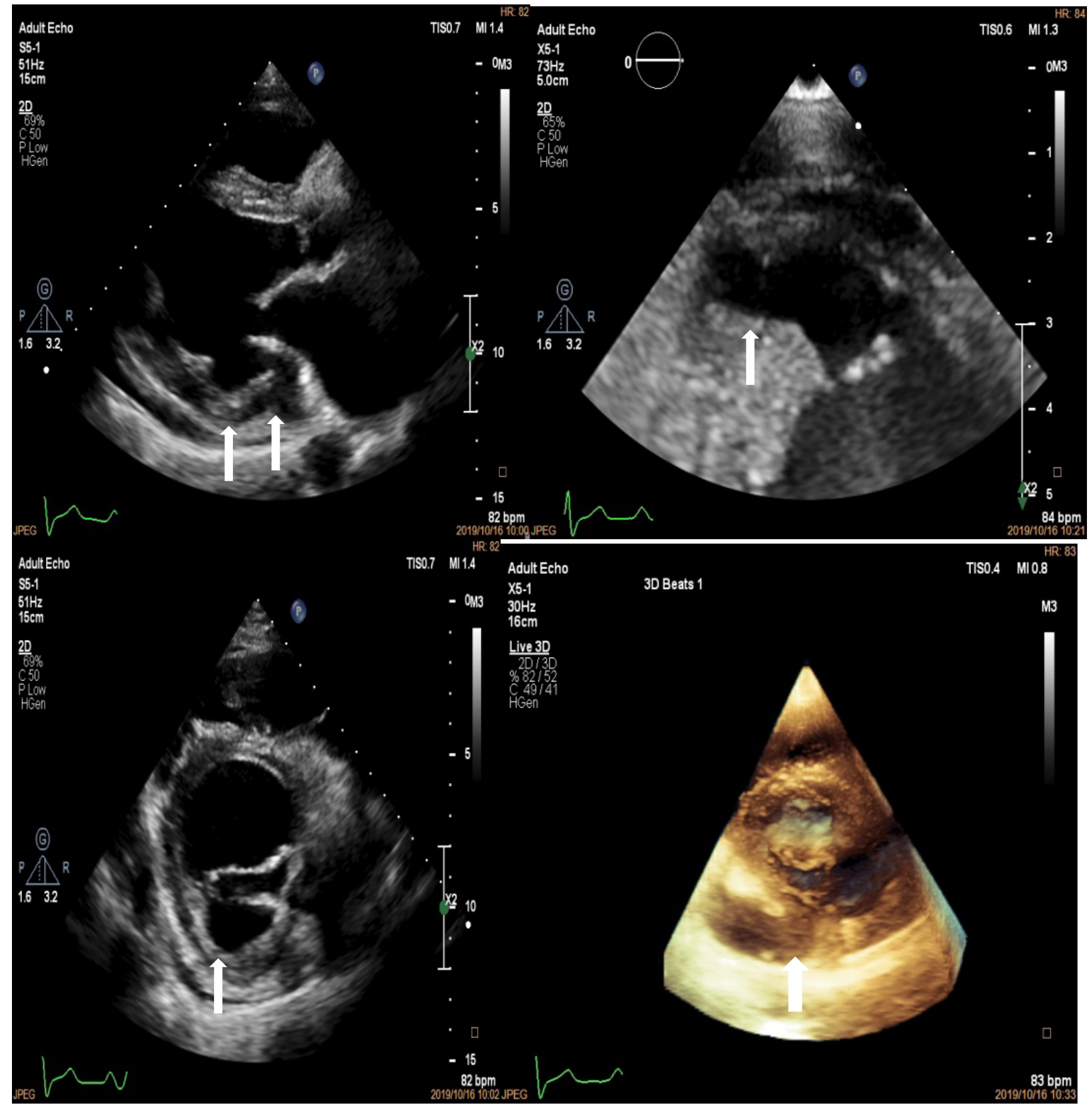

Figure 1: Two dimensional Transthoracic echocardiogram depicting left ventricular aneurysm at the base in the long axis and short axis views (top left and bottom left marked with white arrows), the apex in the zoomed in 4 chamber view (top right, white arrow) and three dimensional echocardiogram showing aneurysm at the base (white arrow). 


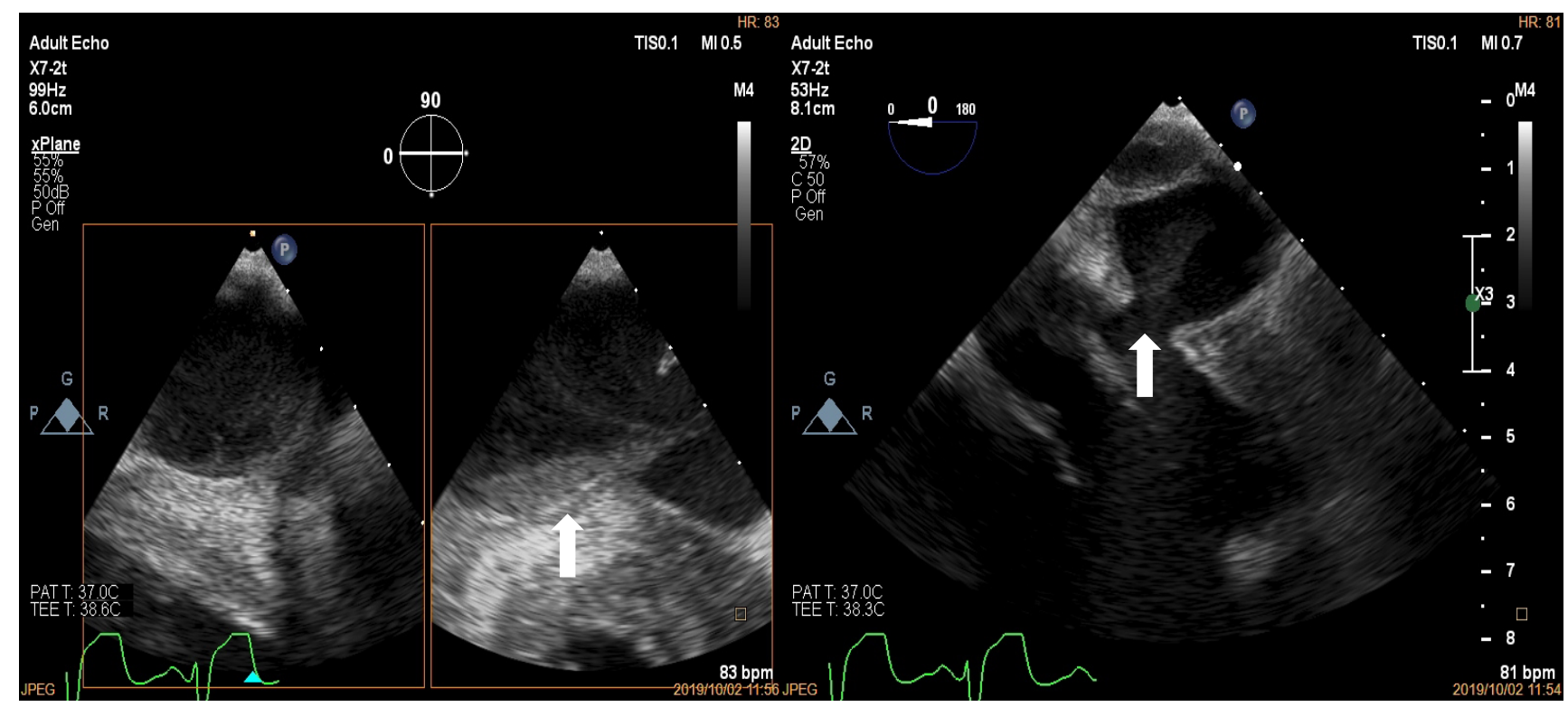

Figure 2: Trans-oesophageal echocardiogram at the mid-oesophageal position showing spontaneous echo contrast and thrombus formation in the sub-mitral aneurysm (white arrows).

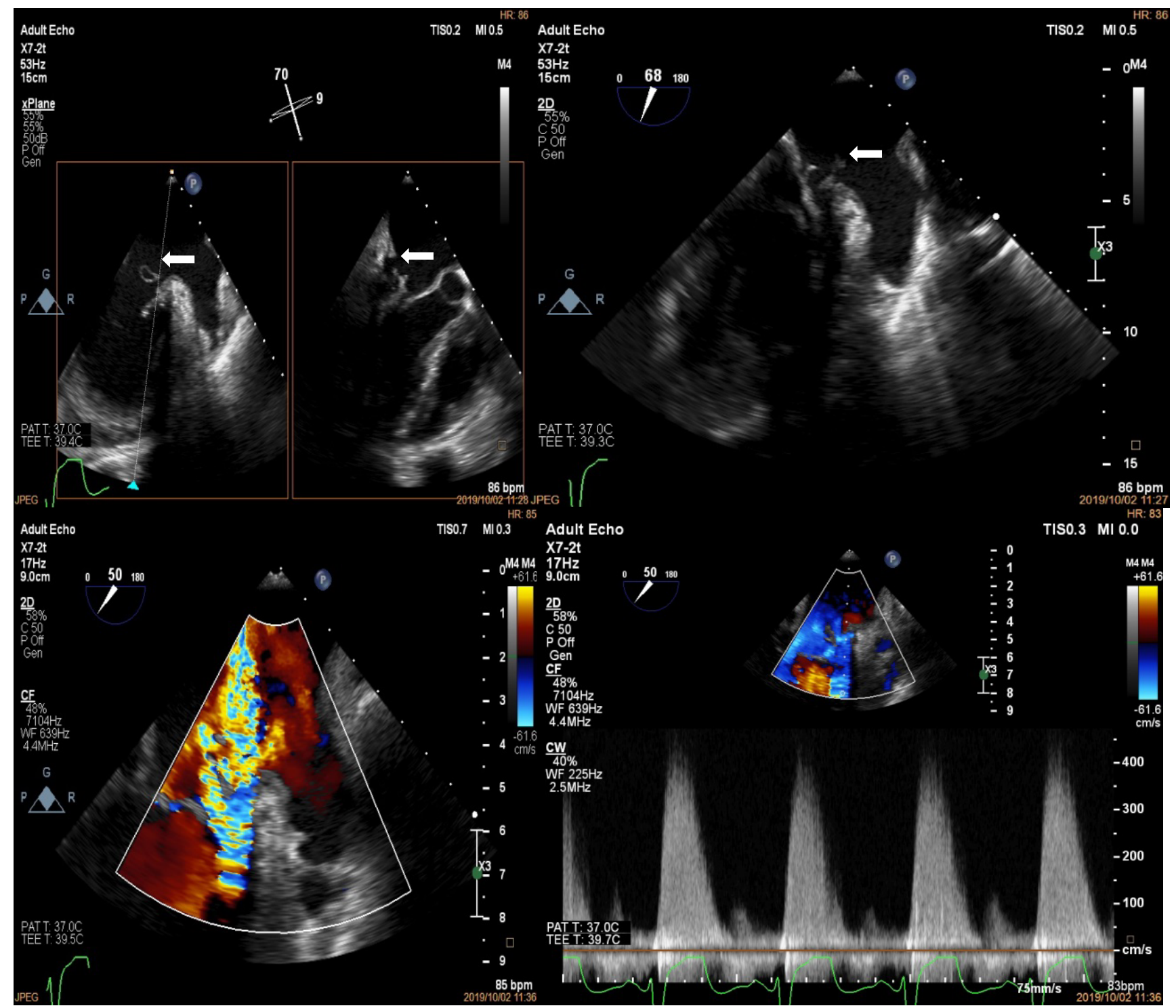

Figure 3: Trans-oesophageal echocardiogram at the mid-oesophageal level showing perforation of the P1 segment of the posterior mitral leaflet (top left, white arrows) with possible vegetation (top right, white arrows). The bottom panels show the severe mitral regurgitation jets on colour (left) and continuous wave Doppler (right). 
posterior mitral leaflet (PML) and the second at the apex of the left ventricle (Figure 1). The patient subsequently underwent two dimensional and three- dimensional transoesophageal echocardiogram (TEE) which con- firmed the presence of sub-mitral aneurysm and apical aneurysm. There was spontaneous echo contrast and thrombus in the sub-mitral aneurysm (Figure 2). The PML was perforated revealing two mitral regurgitation jets,

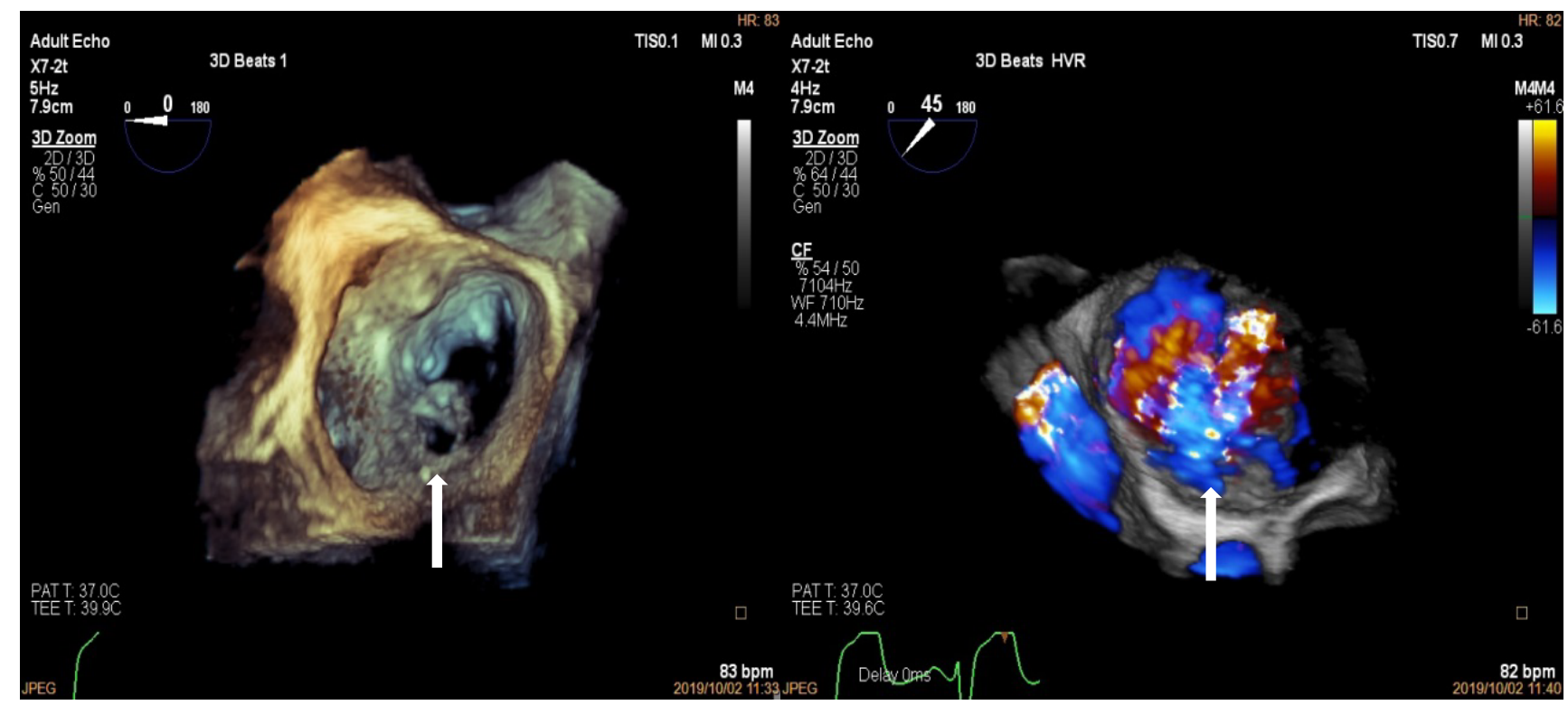

Figure 4: Three-Dimensional Transoesophageal echocardiogram showing "enface view" of the mitral valve with perforation in the P1 segment of the mitral valve (left, white arrow) and three dimensional colour showing mitral regurgitation jet through posterior mitral leaflet perforation (right, white arrow).
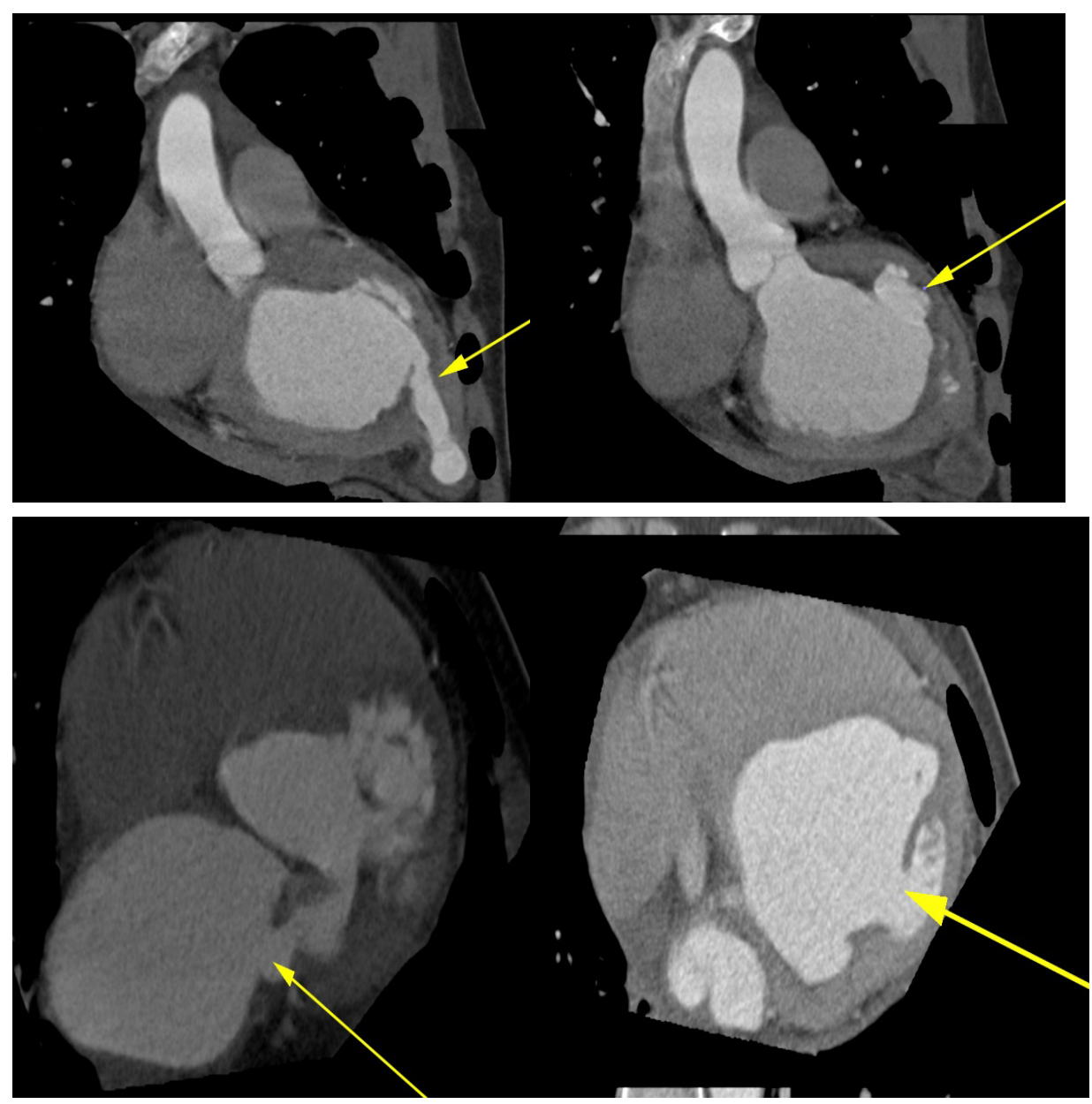

Figure 5: Multi-Detector cardiac computed Tomography showing multiple left ventricular aneurysms at the base, posterior wall, postero-lateral wall and the apex (top and bottom panels, yellow arrows) and perforation in the posterior mitral leaflet (bottom left, yellow arrow). 


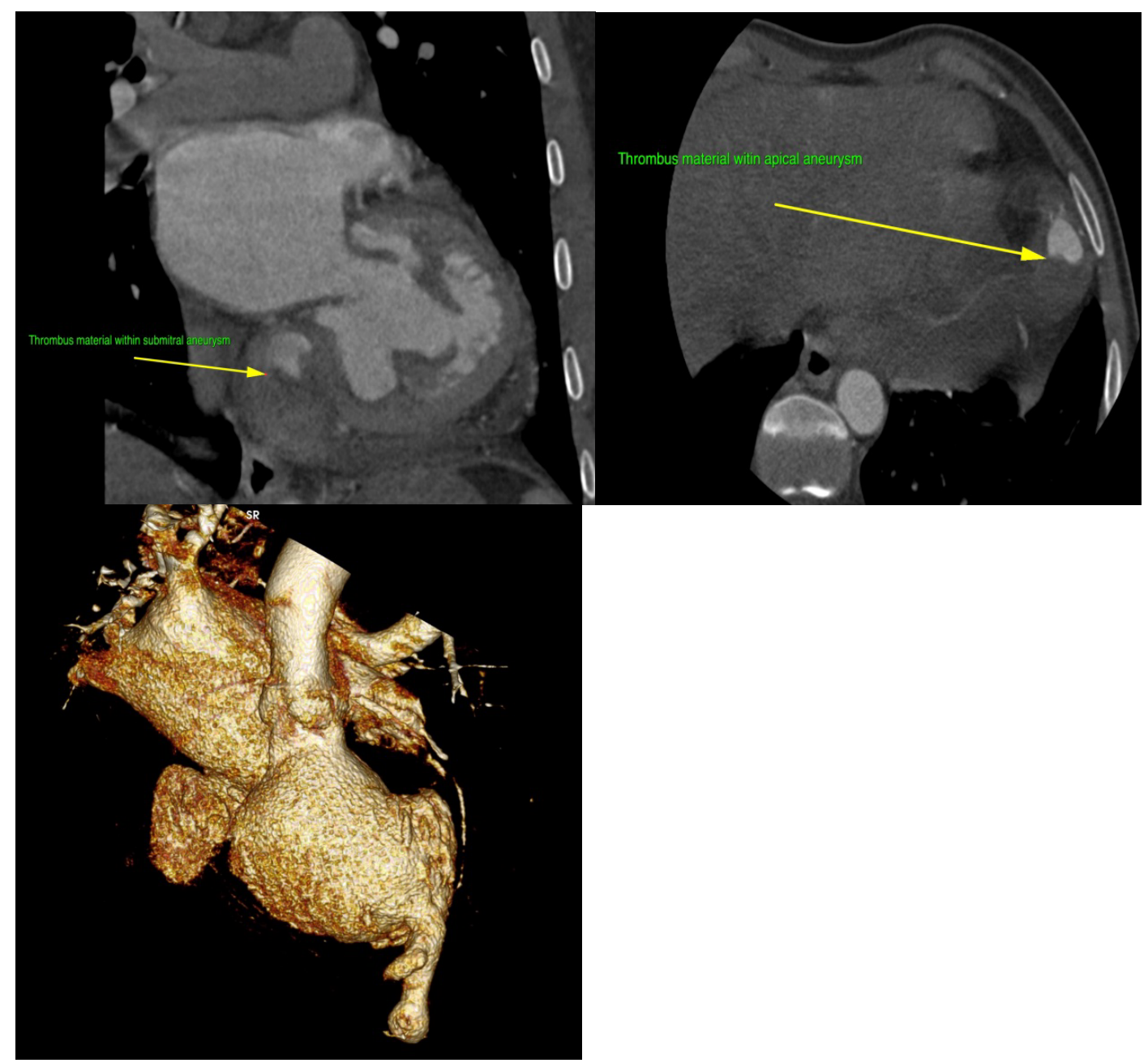

Figure 6: Multi-Detector Cardiac Computed Tomography showing thrombus in the submitral and apical aneurysms (Top panels). Three-dimensional reconstruction (bottom panel) depicting multiple aneurysms of the left ventricle.

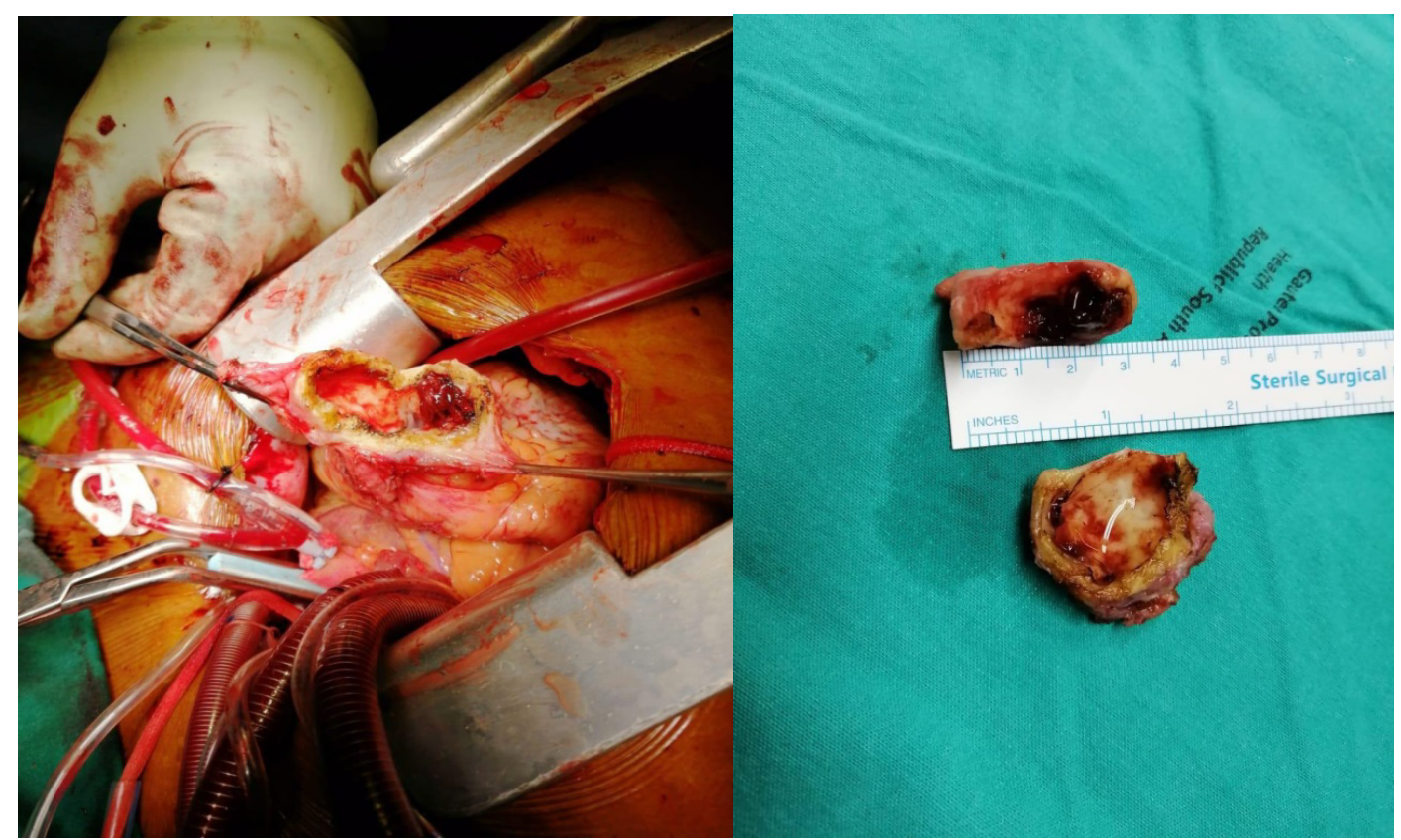

Figure 7: Intraoperative view of the apical aneurysm showing the thick walled aneurysm filled with thrombus (left and right panels).

one through the perforation and the second was central in origin (Figure 3 and Figure 4). There was also suspicion of oscillating mass on the PML (Figure 3). His blood cul- tures remained negative. The gene expert for Tuberculosis was negative. He tested negative for syphilis, Human immunodeficiency virus, connective tissue disease and 
sarcoidosis using the Treponema Haemagglutination test, HIV serology, antinuclear antibody test and serum angiotensin converting enzyme assays, respectively. The patient underwent Multi-detector Computed Tomography (MDCT) to define in detail the anatomical course of the aneurysms and also to exclude coronary artery disease. Multiple left ventricular aneurysms were noted in the posterior wall and the apex (Figure 5 and Figure 6). Aneurysm in the mid-postero-lateral wall measured 38.9 $\mathrm{mm} \times 16.6 \mathrm{~mm}$, sub-mitral aneurysm with thrombus in situ, measured $57.5 \mathrm{~mm} \times 37.8 \mathrm{~mm}$ and it dipped inferior to the left atrium (Figure 6). The apical aneurysm in the inferior aspect was calcified at the antero-lateral margin and measured $54.4 \mathrm{~mm} \times 12.7 \mathrm{~mm}$, and in the septal aspect measured $26.9 \mathrm{~mm} \times 14.7 \mathrm{~mm}$. A discrete perforation was noted in the PML measuring $11.1 \mathrm{~mm}$ in diameter (Figure 5). There was no significant mediastinal lymphadenopathy.

In view of the leaflet perforation and the oscillating mass, patient was started on treatment for probable or partially treated endocarditis due to history of prior antibiotic use, and subsequently underwent urgent surgery. He received mitral valve replacement with plication of sub-mitral aneurysm and partial resection of the apical aneurysm after thrombus removal (Figure 7).

The histology analysis of the valve tissue and the aneurysm showed moderate inflammatory cell infiltrate comprising lymphocytes with focal myxoid areas and dystrophic calcification in the valve and mild chronic inflammation in the aneurysm, also comprising lymphocytes. There were no features of rheumatic fever or infective endocarditis and no granulomas were noted. Culture for Tuberculosis on the myocardial tissue was negative.

A subsequent 18-Flourodeoxyglucose (FDG) Positron emission tomography scan (PET) of the heart was done to exclude possible cardiac sarcoidosis. This test revealed focal uptake of FDG in the basal anterior and basal inferior segments of the myocardium of the left ventricle. The final report was inconclusive and advised correlation with histology and clinical picture.

In view of the histology and the overall clinical picture we concluded that this was likely a congenital or idiopathic disease process. The patient was put on anticoagulation and continued to do well on follow-up. He will be closely watched for future recurrence or expansion of the existing aneurysms.

\section{Discussion}

Multiple intra-cardiac aneurysms are rare [1]. A few case reports have highlighted the presence of such disease process in the African and Asian populations [1-8]. In a large proportion of the patients the aetiology was uncertain and these were classified as congenital or idiopathic. Other differential diagnosis to consider in this case includes sarcoidosis, infective endocarditis, coro- nary artery disease, connective tissue and autoimmune diseases and Tuberculosis. Our case represents first case of multiple cardiac aneurysms with concomitant mitral leaflet perforation in the absence of definite infective endocarditis. We postulate that the primary lesion may have been a mitral leaflet aneurysm analogous to the same aneurysmal disease process in other parts of the left ventricle [9]. Subsequently, the aneurysm in the mitral leaflet likely ruptured and resulted in the patient's presentation with heart failure secondary to severe mitral regurgitation. Second postulate could be a partially treated infective endocarditis at the index admission at the peripheral hospital where he was treated for pneumonia; however, all blood cultures from that admission were sterile.

Initial TTE imaging revealed mitral regurgitation due to prolapse of PML and concurrent sub-mitral aneurysm as well as apical aneurysm, but further definition of the aneurysm extent and presence of vegetation's was not possible. TEE proved useful in further confirming the aneurysms [9]. Three-dimensional TEE was useful in assessing the morphology of the valve and the enface view showed a clear perforation in the P1 segment of the $\mathrm{PML}$ [10]. On multiplane 3D reconstruction the area of the defect in the PML could be measured. Further, two distinct MR jets were visualised on 2D and 3D TEE. One was central due to annular dilation and the second eccentric jet was noted to be coming through the perforation, hence, confirming the presence of a true defect in the mitral valve and not merely an artifact. The suspicion of oscillating mass was raised after TEE imaging due to the superior imaging quality and good temporal resolution of TEE. Finally, MDCT through its good spatial resolution enabled visualisation of the location and anatomy of the aneurysms as well as presence of clear perforation in the PML [11]. It also non-invasively confirmed normal coronary anatomy, thus excluding this as a cause of the left ventricular aneurysms. It established presence of thrombus in the sub-mitral and the apical aneurysm which was not clearly delineated on TEE.

In this case the FDG-PET was suggestive of possible cardiac sarcoidosis. Currently there is no gold standard for the diagnosis of cardiac sarcoidosis [12]. Therefore, the diagnostic performance of FDG-PET is not certain. True assessment of isolated cardiac sarcoidosis in the absence of systemic disease as in this case is even more challenging. An assessment of possible sarcoidosis on FDG-PET implies less than $50 \%$ probability of patient having cardiac sarcoidosis and therefore we deemed it suitable to not start treatment with steroids and decided to watch the patient closely.

This case illustrates the value of multimodality imaging of a complex case prior to surgical intervention. It also brings to the fore a multitude of differential diagnosis that one must consider when evaluating a patient with ventricular aneurysms. 


\section{Conflicts of Interest}

None.

\section{Funding}

The first author was the recipient of the Post-Doctoral Carnegie Fellowship award.

\section{References}

1. Harris Jr GA, Wong AK, Lantz DA (1999) Multiple idiopathic left ventricular aneurysms in a Japanese woman. Military Medicine 164: 448-450.

2. Chesler E, Joffe N, Schamroth L, Meyers A (1965) Annular subvalvular left ventricular aneurysms in the South African Bantu. Circulation 32: 43-51.

3. Okuwobi BO (1975) Multiple ventricular aneurysms. The American Journal of Cardiology 36: 521-523.

4. Bennett JM, Naude DF, De Villiers JS (1989) Recurrence of two myocardial aneurysms infected with tuberculosis after a previous aneurysmectomy. Clinical Cardiology 12: 605606.

5. Gokhale AG, Lal N, Ashok B, Jacob J, Krishnaswami S, et al. (1993) Mitral valve replacement for an annular submitral aneurysm of the left ventricle. Thorax 48: 676-677.

6. Fitchett DH, Kanji M (1983) Mitral subannular left ventricu- lar aneurysm: A case presenting with ventricular tachycardia. Br Heart J 50: 594-596.

7. Paul M, Schäfers $M$, Grude $M$, Reinke F, Juergens KU, et al. (2006) Idiopathic left ventricular aneurysm and sudden cardiac death in young adults. Europace 8: 607-612.

8. Ouyang F, Antz M, Deger FT, Bänsch D, Schaumann A, et al. (2003) An underrecognized subepicardial reentrant ventricular tachycardia attributable to left ventricular aneurysm in patients with normal coronary arteriograms. Circulation 107: 2702-2709.

9. Tariq M, Zahid I, Sami S (2019) Rare aneurysm of anterior mitral valve leaflet-a case report. Journal of Cardiothoracic Surgery 14: 1-4.

10. Kharwar RB, Mohanty A, Sharma A, Narain VS, Sethi R (2014) Ruptured anterior mitral leaflet aneurysm in aortic valve infective endocarditis--evaluation by three-dimensional echocardiography. Echocardiography 31: E72-E76.

11. Kim JH, Kim EY, Jin GY, Choi JB (2017) A review of the use of cardiac computed tomography for evaluating the mitral valve before and after mitral valve repair. Korean J Radiol 18: 773-785.

12. Chareonthaitawee $P$, Beanlands RS, Chen W, Dorbala S, Miller EJ, et al. (2017) Joint SNMMI-ASNC expert consensus document on the role of 18F-FDG PET/CT in cardiac sarcoid detection and therapy monitoring. $\mathrm{J}$ Nucl Med 58: 1341-1353. 